\title{
Sport und Bewegung mit und nach Krebs - wer profitiert, was ist gesichert?
}

\author{
Exercise and Physical Activity During and after Cancer - Who \\ Benefits most, What is Proven?
}

\author{
Autoren \\ Karen Steindorf', Martina E. Schmidt' ${ }^{1}$, Philipp Zimmer ${ }^{1,2}$ \\ Institute \\ 1 Deutsches Krebsforschungszentrum (DKFZ) Heidelberg \\ 2 Institut für Kreislaufforschung und Sportmedizin, \\ Deutsche Sporthochschule Köln \\ Schlüsselwörter \\ Sport, Bewegung, Krebs, Überleben, Nebenwirkungen
}

Key words

exercise, physical activity, cancer, survival, side effects

Bibliografie

DOI https://doi.org/10.1055/a-0596-8629

TumorDiagn u Ther 2018; 143: 301-306

(c) Georg Thieme Verlag KG, Stuttgart · New York

ISSN 0722-219X

Korrespondenzadresse

Prof. Dr. Karen Steindorf

Abteilung Bewegung, Präventionsforschung und Krebs

(G210)

Deutsches Krebsforschungszentrum (DKFZ) und Nationales

Centrum für Tumorerkrankungen (NCT), Im Neuenheimer

Feld 280, 69120 Heidelberg

k.steindorf@dkfz.de

\section{ZUSAMMENFASSUNG}

Die Diagnose „Krebs“ und die anschließende medizinische Therapie verleiten viele Patienten dazu ihre körperlichen Aktivitäten einzuschränken. Doch gerade Sport und bewegungstherapeutische Programme sind effektive supportive Maßnahmen im Kampf gegen den Krebs und manche Nebenwirkungen. Zudem haben die Betroffenen eine einzigartige Chance, selbst aktiv an ihrem Genesungsprozess mitzuarbeiten. Was zu berücksichtigen ist, zeigt dieser Beitrag.

\section{ABSTRACT}

A growing number of literature reports positive effects of physical activity and exercise. These effects concern prevention, mortality and progress of cancer. Furthermore they show a reduction of disease- and treatment-specific side effects, which implies a better patients' quality of life. Evidence shows a decreased mortality risk in patients with physically active cancer of colon, breast, ovaries and prostate. Activity recommendations for patients with cancer do not differ from those for healthy persons. However, a decrease in physical activity can be observed in many cancer patients after diagnosis, and this fact concludes to a reduced physical capacity although just physical capacity helps to overcome the burden of medical treatment and to recover. In fact, tailored exercise programs can counteract a decrease in physical capacity successfully. Further they reduce side effects such as fatigue, incontinence and lymphedema. Moreover, first studies report a positive influence on: chemotherapy-induced polyneuropathies, cancer-related cognitive impairments, bone health and sleep. Finally, an enormous number of investigations showed that exercise interventions can improve psychosocial factors (e.g. mood and self-esteem). Except for very few conditions, such as extremely impaired blood cell counts and days when cardio- or nephrotoxic drugs are given, physical activity should be recommended. Especially during medical treatment, physicians and therapists are prompted to interact closely in order to prevent general activity prohibitions. Physical activity and exercise programs represent a unique and low-cost chance for cancer patients to influence the course of their own disease positively. More effort is necessary to improve professional activity offers for cancer patients and survivors and to specify activity recommendations.
Sport und körperliche Aktivität haben einen positiven Einfluss auf den Verlauf zahlreicher Tumorerkrankungen und deren Nebenwirkungen. Immer mehr Interventionsstudien belegen dies. Je nach Belastungsmodalität (Art, Dauer, Intensität und Häufigkeit) der körperlichen Aktivität kommt es zu einer Vielzahl von physiologi- schen und psychologischen Adaptionen. Bewegung und Sport wirken jedoch nicht nur lokal, also in der beanspruchten Muskulatur, sondern haben auch systemische Effekte. Körperliches Training wird mittlerweile mit einem breiten Wirkspektrum in Verbindung gebracht ( $\triangleright$ Abb. 1$)$ : 




- Abb.1 Die Bedeutung von körperlicher Aktivität im Kontext onkologischer Erkrankungen.

- Nebenwirkungen des Tumors oder der Therapie werden verhindert bzw. gelindert,

- dadurch werden Therapieverträglichkeit und Wirksamkeit verbessert, und damit auch

- die Prognose (Rezidivhäufigkeit, Mortalität).

Aufgrund von Ergebnissen tierexperimenteller Studien wird in jüngster Zeit sogar diskutiert, ob Sport eine direkte antitumorale Wirkung haben kann.

\footnotetext{
Merke

Laut Definition umfasst körperliche Aktivität das gesamte Bewegungsverhalten im Beruf, im Alltag und in der Freizeit. Sport als gezieltes Training ist dabei eine Möglichkeit, körperlich aktiv zu sein.
}

\section{Körperliche Aktivität mindert Risiken}

Große epidemiologische Beobachtungsstudien zeigen: Ein erhöhtes körperliches Aktivitätsniveau

- wirkt nicht nur der Entstehung von Tumorerkrankungen entgegen [1], sondern

- ist auch bei bestehenden Tumorerkrankungen mit einer Reduktion des krebsspezifischen Risikos und des Gesamtmortalitätsrisikos assoziiert [2].

Dies gilt auch, wenn das Bewegungsverhalten erst nach der Diagnose gesteigert wird. Außerdem weisen erste Studienergebnisse darauf hin, dass sich eine höhere körperliche Aktivität mindernd auf die Rezidiv-Wahrscheinlichkeit auswirkt [2].

Die beste Evidenzlage zum Einfluss des Bewegungsverhaltens auf das Mortalitätsrisiko liegt bislang für Patienten mit Brustoder Darmkrebs vor. So kann das Risiko, an einer Darmkrebserkrankung zu versterben, durch eine gesteigerte körperliche Aktivität um bis zu $40 \%$ reduziert werden [3].
Merke

Wenngleich dieser Effekt beachtlich ist, gilt: Bewegung und Sport können keine schulmedizinische Therapie ersetzen, sondern sollten als effiziente Supportivmaßnahmen verstanden werden.

\section{Mechanismen}

Die biologischen Hintergründe dieser Zusammenhänge und weiterer positiver Effekte von körperlicher Aktivität sind bislang nur rudimentär verstanden. Grundsätzlich lässt sich sagen: eine regelmäßige körperliche Aktivität trägt über

- eine Reduktion der Fettmasse und

- eine Aktivierung bestimmter Immunzellen (z. B. regulatorischer T-Lymphozyten)

dazu bei, einer systemischen Entzündung entgegenzuwirken [4]. Vor allem diese systemische Entzündung, die mit steigendem Lebensalter zunimmt, scheint ein wichtiger Risikofaktor für die Entstehung und den Progress vieler Tumorerkrankungen zu sein.

Intensivere körperliche Betätigungen können die endogene Tumorabwehr aktivieren. Durch akute Belastungen werden beispielsweise natürliche Killerzellen mobilisiert und aktiviert. Aktuelle tierexperimentelle Arbeiten belegen: Die Immunzellen, die Tumorzellen erkennen und abtöten können, sind nach einem Trainingsprogramm tatsächlich

- vermehrt in Tumoren aufzufinden und

- direkt mit einer verzögerten Tumorentstehung und einem reduzierten Progress assoziiert [4].

\section{Merke}

Sowohl die Abwehr der systemischen Inflammation als auch die endogene Tumorabwehr wirken sich aller Wahrscheinlichkeit nach bei vielen neoplastischen Erkrankungen positiv aus.

Es ist nicht auszuschließen, dass körperliche Aktivität bestimmte Krebsarten auch über andere Wege beeinflusst (z. B. hormonsensitive Tumorerkrankungen wie Prostata- oder Brustkrebs über die Veränderung endokriner Regelkreise). Hier herrscht wissenschaftlicher Forschungsbedarf, um in Kombination mit klinischen Studien künftig eine Basis für konkrete Bewegungsempfehlungen (Art, Dauer, Intensität, Frequenz) formulieren zu können.

\section{Körperliche Aktivität mindert Neben- wirkungen}

Insbesondere gezielte Bewegungsprogramme können effizient helfen, Nebenwirkungen onkologischer Erkrankungen und deren Therapien zu reduzieren. Neben dem besseren Wohlbefinden der Betroffenen hat dies eine hohe klinische Relevanz: Nebenwirkungen schränken häufig die Therapiedosis ein und damit auch die Wirksamkeit. Im Folgenden werden die aktuellen Erkenntnisse zu einigen häufig auftretenden Nebenwirkungen dargelegt. Die diesbezügliche Studienlage betrifft vor allem den Brustkrebs. Es ist jedoch davon auszugehen, dass viele Fakten auch für andere Entitäten gelten, insbesondere, wenn dort vergleichbare Therapieformen angewendet werden. 


\section{Fatigue}

Mit $60-80 \%$ ist das Fatigue-Syndrom eine der häufigsten Nebenwirkungen bei Krebserkrankten. Zudem beeinträchtigt es die Patienten oft noch Jahre nach der Therapie. Im Kontext von Bewegungsinterventionen und Tumorerkrankungen ist dementsprechend Fatigue auch die am meisten untersuchte Nebenwirkung.

\section{Merke}

Bislang sind keine medikamentösen oder supportiven Maßnahmen bekannt, die dem Fatigue-Syndrom so effizient entgegenwirken können wie Sport bzw. gezielte bewegungstherapeutische Programme [5, 6].

Neuere Arbeiten konnten zeigen: Bewegungsinterventionen sollten simultan zur medizinischen Therapie stattfinden, um möglichst früh im Behandlungsverlauf aktiv der Entstehung von Fatigue entgegenzuwirken. Neben einer Chemo- oder Strahlentherapie ist das Krafttraining anderen supervidierten Supportivmaßnahmen wie z. B. einem Entspannungstraining überlegen $[7,8]$.

Bislang wird vermutet, dass die positiven Effekte von Bewegung und Sport auf das Fatigue-Syndrom sowohl auf physiologischer als auch auf psychologischer Ebene stattfinden. Neben der bereits o. g. Wirkung von Bewegung auf den Inflammationsstatus wird auch die Beeinflussung von Hormonen und Hormonachsen (z.B. Cortisol) diskutiert. Zentrale Aufgabe zukünftiger Studien wird zum einen sein, konkretere Bewegungsempfehlungen für verschiedene Ausprägungen des Fatigue-Syndroms zu formulieren. Zum anderen ist der Einfluss verwandter Nebenwirkungen (z.B. Depressionen, kognitive Beeinträchtigungen) noch besser herauszuarbeiten.

\section{Merke \\ Allein die derzeitigen Erkenntnisse zu den positiven Effekten von Bewegung zur Verhinderung oder Linderung von Fatigue sollten ausreichend sein, um körperliche Aktivität zu einem festen Bestandteil der supportiven Krebstherapie zu machen.}

\section{Harninkontinenz}

Nach krankheitsbedingten Eingriffen im Urogenital-Trakt wird die Kontinenz durch bewegungstherapeutische Maßnahmen, insbesondere durch ein Schließmuskel-/Beckenbodentraining nicht nur schneller, sondern auch vollständiger wiedererlangt. Dies zeigen mehrere Untersuchungen. Zudem ist ein solches Training unter Anleitung eines Therapeuten deutlich effizienter als ohne Supervision [9]. Erste Studien beschreiben, dass es sogar ratsam ist, im Sinne einer Prähabilitation schon vor der geplanten medizinischen Therapie (z.B. Prostataresektion) mit dem Training zu beginnen. Eine randomisierte kontrollierte Studie konnte nachweisen, dass ein Beckenbodentraining mit präoperativem Beginn zu einer deutlich schnelleren Rekonvaleszenz der Kontinenz führt [10]. Da sich eine persistierende Inkontinenz überaus negativ auf die Lebensqualität auswirkt, sollte der Patient umfassend über bewegungstherapeutische Möglichkeiten aufgeklärt werden.

\section{Lymphödem}

Lange Zeit wurde Patienten mit Lymphödemen (häufig ArmLymphödeme nach operativer Entnahme von Lymphknoten und Bestrahlung) angeraten, die betroffene Struktur zu schonen. Inzwischen ist jedoch nachgewiesen: Patienten profitieren von einem gezielten (Kraft-) Training, gerade auch der betroffenen Extremität. So lassen sich sowohl das Lymphödem als auch die damit verbundenen Problematiken wie Schmerzen und Sensibilitätsausfälle verringern [11].

\section{Polyneuropathie}

Erste Studien weisen darauf hin, dass multimodale Bewegungsprogramme mit sensomotorischen Trainingselementen das Potenzial haben, der Entstehung und dem Schweregrad einer Chemotherapie-induzierten Polyneuropathie entgegenzuwirken [12].

\section{Abnahme der Hirnleistung}

Tumorerkrankungen und deren medizinische Therapien können zu Einbußen der Hirnleistungsfähigkeit führen (früher als „Chemobrain“ bezeichnet). Neben strukturellen Veränderungen treten bei Patienten häufig Konzentrations- und Erinnerungsprobleme auf - teilweise auch noch Jahre nach der Therapie.

\section{Merke}

Es gilt als gesichert, dass regelmäßige körperliche Aktivität und v. a. Ausdauerbelastungen sowohl strukturell als auch funktionell die Hirnleistung positiv beeinflussen können und darüber hinaus neuroprotektive Effekte haben.

Dennoch ist die Datenlage bezüglich krebsassoziierter kognitiver Beeinträchtigungen wenig aussagekräftig [13]. Ganz ähnlich wie bei den Polyneuropathien sind hier in naher Zukunft von laufenden wissenschaftlichen Studien genauere Aussagen zu erwarten.

\section{Kachexie}

Da besonders Krafttraining zu Muskelerhalt und -aufbau führt, liegt die Vermutung nahe, dass man durch gezielte Krafttrainingsinterventionen einer Tumorkachexie vorbeugen könnte. Hier stehen jedoch belastbare Studienergebnisse aus.

\section{Knochenveränderungen}

Ebenfalls diskutiert wird, inwieweit gezielte Trainingsinterventionen die Knochengesundheit von Krebspatienten positiv beeinflussen können. Neben therapiebedingten osteoporotischen Veränderungen stellen Knochenmetastasen ein erhebliches Problem dar, insbesondere, wenn diese frakturgefährdend sind. Wenn aber keine akute Frakturgefährdung vorliegt, kann eine adäquate Belastung der Knochenstruktur zu einer Stärkung der Knochensubstanz führen [14]. Allerdings liegt hier bislang keine ausreichende Evidenz für Bewegungsempfehlungen vor. 


\section{Schlafprobleme}

Schlafprobleme sind eine weitere häufig auftretende und belastende Symptomatik für viele Krebsbetroffene. Die Linderung von Schlafproblemen durch körperliches Training konnte in neueren Studien belegt werden [15].

Merke

Gezielte Bewegungsprogramme haben das Potenzial, viele relevante Nebenwirkungen von Krebserkrankungen und deren Therapie zu verringern.

\section{Körperliche Aktivität steigert Lebens- qualität}

Drei große Übersichtsarbeiten beschäftigten sich mit dem Einfluss von Bewegung auf die Lebensqualität von Tumorpatienten. Ergebnis dieser Studien:

\section{Merke}

Tumorpatienten, die während und nach der medizinischen Therapie an Bewegungsprogrammen teilnehmen, weisen eine Lebensqualität auf, die im Schnitt $48 \%$ über der von Kontrollgruppenpatienten liegt [16 - 18].

Nicht nur die Effekte auf Fatigue spielen hier eine Rolle, sondern auch die durch das Training verbesserte körperliche Leistungsfähigkeit. Insofern wirken Bewegungsprogramme auch dem Umstand entgegen, dass diagnose- und therapiebedingt viele Patienten ihr Aktivitätsniveau drastisch verringern - ein Verhalten, das ja auch bei gesunden Menschen zur Abnahme der Fitness führt.

\section{Psychosoziale Effekte}

Häufig haben Bewegungsinterventionen nicht nur positive körperliche, sondern auch positive psychosoziale Effekte. Diese tragen letztlich auch zu einer verbesserten Lebensqualität bei. Im Vordergrund stehen dabei folgende Faktoren:

- Strukturierung des Alltags

- Abwenden einer evtl. drohenden sozialen Isolation

- verbessertes Selbst- und Körperbild

- mögliche Reduktion von Depressionen und Ängsten

- Autonomie erhalten oder zurückgewinnen

- das generell als positiv empfundene Gefühl, selbst zum Therapieerfolg beizutragen

\section{Praktische Empfehlungen}

\section{Wieviel Aktivität?}

Die aktuellen Empfehlungen besagen: Krebsbetroffene sollen über den gesamten Krankheits- und Behandlungszeitraum so früh wie möglich so aktiv wie möglich sein. Anzustreben sind pro Woche mindestens 150 Minuten moderate oder 75 Minuten intensive körperliche Aktivität. Neben Ausdauerbelastungen wird auch zu einem regelmäßigen Krafttraining (2-3-mal/Woche) geraten [19].

\section{Merke}

Damit ist die Empfehlung für Krebsbetroffene im Grundsatz identisch mit den Bewegungsempfehlungen für die allgemeine Bevölkerung.

Natürlich stellt sich die Situation im konkreten Fall meist komplexer dar. Das Training ist kontinuierlich anzupassen an

- die individuelle Leistungsfähigkeit,

- spezielle Aspekte der Erkrankung,

- die Therapieform (z. B. Tragen eines Stomas) und

- spezifische Bedürfnisse und Möglichkeiten des Patienten.

\section{Wie wird trainiert?}

Die Art des Trainings wird in der Richtlinie wenig spezifiziert. Maßgeblich sind das Trainingsziel und die individuelle Situation des Patienten. Dabei kann sich der Fokus des Trainings über die gesamte Zeit durchaus ändern. Es sind einige Aspekte zu berücksichtigen:

- Geht es um die Erhaltung der Knochengesundheit, sind andere Trainingsreize zu setzen als z. B. bei der Behandlung oder Prävention von Polyneuropathien oder Lymphödemen.

- Individuelle (1:1) Bewegungsangebote mit einem Trainer und einem Patienten können erforderlich sein, so z. B. in der Isolationsphase während der Therapie einer hämatoonkologischen Erkrankung.

- In einer anderen Situation würde man dagegen möglicherweise gezielt zu Gruppenangeboten raten oder zur Einbindung von nahestehenden Personen.

- Patienten, die sich außerhalb von supervidierten Trainingsinterventionen intensiver belasten möchten, wird zu einer Sporttauglichkeitstestung bei einem Facharzt geraten. Meist werden die anfallenden Kosten von den Kassen übernommen.

- Wichtig ist zudem, dass die Bewegungsmaßnahmen Freude bereiten, gerade auch im Sinne der Nachhaltigkeit.

Merke

Grundsätzlich sollte so früh wie möglich nach der Diagnosestellung auf ein hinreichendes Aktivitätsniveau geachtet werden.

\section{Kontraindikationen}

Fast alle Tumorpatienten profitieren von Bewegung und Sport. Dennoch gibt es während des Krankheits- und Therapieverlaufs Phasen, in denen Patienten sich nicht oder nur wenig belasten sollten. Generelle Bewegungsverbote auszusprechen ist in den allermeisten Fällen problematisch, da eine Immobilisation zu weitreichenden physischen und psychosozialen Konsequenzen führen kann. Im Übrigen gelten auch für Krebskranke die allgemeinen Kontraindikationen in Bezug auf akute körperliche Betätigungen: Infektionen (v.a. febril), starke Schmerzen, starke Übelkeit und Schwindel.

\section{Blutwerte}

Vor allem unter Chemo- und Strahlentherapie können Blutwerte auftreten, die eine körperliche Betätigung nicht mehr zulassen, 
wie z. B. sehr niedrige Hämoglobinwerte. Hierzu fehlen allerdings einheitliche und aktuelle Empfehlungen. 1986 publizierten Winningham et al. Grenzwerte, die heute nur noch eingeschränkte

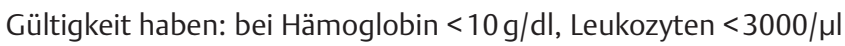


indiziert [20]. Elter et al. berichteten 2009, dass auch mit deutlich niedrigeren Werten zumindest ein Ausdauertraining noch gut möglich ist. Ferner wiesen sie darauf hin: Durch zu hoch angesetzte Grenzwerte können viele Patienten unter Therapie nicht von den positiven Effekten gezielter Bewegungsprogramme profitieren [21].

\section{Knochenmetastasen}

Spezielle Kontraindikationen für Krebspatienten sind in aller Regel Einzelfallentscheidungen, wie z. B. bei frakturgefährdenden Metastasen in der Wirbelsäule. Knochenmetastasen, die nicht frakturgefährdend sind, stellen keine Kontraindikation dar. Im Gegenteil: Hier würde ein Bewegungsverbot zu einer weiteren Schwächung der Knochensubstanz führen - bis hin zu osteoporotischen Veränderungen, die letztlich eine Fraktur begünstigen. Entsprechend ist Betroffenen ein gezieltes Training zur Stärkung der Struktur zu empfehlen.

\section{Eingeschränkte Kontraindikationen}

Darüber hinaus sollte an Tagen, an denen bestimmte medizinische Therapien (z. B. kardio- oder nephrotoxische Chemotherapien) angesetzt sind, keine intensive körperliche Belastung stattfinden, d. h. 24 Stunden kein Sport. Diese Empfehlung ist derzeit nicht evidenzbasiert, sondern gilt als reine Vorsichtsmaßnahme, da intensive körperliche Betätigung auch die genannten Organe fordert. Operationsnarben, Knochenmetastasen (s. o.) und das Tragen eines Stomas sind keine generellen Kontraindikationen. Trotzdem sollten Bewegungsprogramme in diesen Fällen nach Rücksprache mit einem fachkundigen Arzt, Physio- oder Sporttherapeuten geplant und/oder durchgeführt werden [22].

\section{Merke}

Bei onkologischen Patienten gibt es weniger Kontraindikationen für körperliches Training als vielfach angenommen. Dennoch setzt das Durchführen der Trainingsmaßnahmen, v. a. unter Therapie, oft ein spezielles Know-how voraus.

\section{Versorgungssituation}

\section{Ärztliche Beratung}

Das Feld „Bewegung, Sport und Krebs“ ist ein interdisziplinäres Thema, in dem i.d.R. keiner Fachkraft per se das volle Wissensspektrum zur Verfügung steht. Viele Ärzte fühlen sich nicht hinreichend kompetent für eine gute Beratung. Zudem verweisen sie oft auf den begrenzten Zeitrahmen und zu geringe Abrechnungsmöglichkeiten für beratende Tätigkeiten. Dennoch stellen Ärzte gemäß ihrem Versorgungsauftrag sowie aus Patientensicht un- zweifelhaft eine wichtige Schnittstelle dar. Den Patienten auf die positiven Effekte von Bewegung aufmerksam zu machen, sollte als Minimum angesehen werden.

\section{Strukturen}

Darüber hinaus gilt es, aktuellen Beratungs- und Versorgungsengpässen zu begegnen. Hierzu sind bessere Allianzen erforderlich zwischen

- den einzelnen bewegungstherapeutischen Facheinrichtungen (Rehabilitations- und Gesundheitszentren, Physiotherapiepraxen, sportmedizinische Einrichtungen),

- onkologischen oder hausärztlich ausgerichteten Praxen und Fachzentren,

- Selbsthilfegruppen, Vereinen und

- relevanten Fachgesellschaften.

\section{Merke}

Auch die Fort- und Weiterbildungsangebote für Ärzte und das Pflegepersonal sowie für Physiotherapeuten sind zu verbessern.

Eine flächendeckende Beratungs- und Versorgungsstruktur, die allen Krebsbetroffenen ein bedarfsgerechtes und wohnortnahes körperliches Training ermöglicht, ist in Deutschland bislang nicht vorhanden. Andererseits findet eine stetige Professionalisierung und Ausweitung der Sport- und Bewegungsangebote speziell für onkologische Patienten statt [22]. Dieser Fortschritt bedarf unbedingt weiterer struktureller Förderung; denn demografisch bedingt wird die Zahl der Krebsbetroffenen in Deutschland steigen und damit auch die Wahrnehmung des Themas in der Öffentlichkeit. Es gibt bereits auf das Personal ausgerichtete Fortbildungsangebote. Außerdem sollten aber auch Maßnahmen zur Qualitätssicherung (z. B. Zertifizierungen) den Standard von Trainingseinrichtungen und -angeboten speziell für Krebsbetroffene gewährleisten.

\section{Sport auf Rezept?}

Eine flächendeckende onkologische Sport- und Bewegungstherapie wird bislang durch das deutsche Gesundheitssystem nicht finanziert. Doch gibt es erfolgreiche Pilotprojekte, die zeigen, dass hier in Kürze Veränderungen zu erwarten sind. Schließlich werden von den Krankenkassen Medikamente gegen eine bestimmte Nebenwirkung bei Vorliegen eines Wirksamkeitsnachweises finanziert. Warum dann nicht auch das Fördern von körperlicher Aktivität in der Krebstherapie? Diese Frage ist insbesondere für das Fatigue-Syndrom berechtigt: Schließlich ist hier bislang für kein Medikament eine überzeugende Wirksamkeit nachgewiesen worden. Darüber hinaus haben Betroffene laut Sozialgesetzbuch IX §44 ein Anrecht auf Rehabilitationsmaßnahmen. So wird beispielsweise die Teilnahme an Krebssportgruppen mit bis zu 6,40 Euro pro Einheit unterstützt. Der behandelnde Arzt muss dafür lediglich das Musterformular 56 ausfüllen (siehe Homepage der Kassenärztlichen Bundesvereinigung). 


\section{INFOBOX}

Weiterführende Weblinks

- https://www.nct-heidelberg.de/fileadmin/media/fuer_ patienten/beratung/bewegung/OnkoAktiv/ 0706_Sport_und_Krebs_web.pdf

- https://www.krebshilfe.de/fileadmin/Downloads/PDFs/ Blaue_Ratgeber/048_0046.pdf

\section{KERNAUSSAGEN}

- Ein gesteigertes Aktivitätsniveau reduziert das Krebsrisiko.

- Ein gesteigertes Aktivitätsniveau reduziert das Sterberisiko bei zahlreichen Krebserkrankungen.

- Gezielte Bewegungsprogramme reduzieren häufig auftretende Nebenwirkungen der Erkrankung und deren Therapie (z. B. Fatigue-Syndrom).

- Die Teilnahme an Bewegungsprogrammen steigert während und nach der Therapie die Lebensqualität.

- Bewegung und Sport bieten Betroffenen eine einzigartige Möglichkeit, ihren Krankheitsverlauf selbst positiv zu beeinflussen und aktiv zur Genesung beizutragen.

- Ein Mindestmaß an Aufklärung sollte keinem Patienten vorenthalten werden.

- Eine weitere Professionalisierung und Vernetzung aller Beteiligten (Ärzte, Therapeuten, Pflegepersonal, Wissenschaftler, Kassen etc.) ist notwendig, um die Versorgungssituation für Betroffene zu verbessern.

\section{Interessenkonflikt}

Die Autoren geben an, dass kein Interessenkonflikt besteht.

\section{Erstveröffentlichung}

Dieser Beitrag wurde erstveröffentlicht in: Dtsch Med Wochenschr 2018; 143: 309-315.

Literatur

[1] Leitzmann M, Powers H, Anderson AS et al. European Code against Cancer 4th Edition: Physical activity and cancer. Cancer Epidemiol 2015; 39 (1): S46-S55. doi:10.1016/j.canep.2015.03.009

[2] Cormie P, Zopf EM, Zhang X et al. The Impact of Exercise on Cancer Mortality, Recurrence, and Treatment-Related Adverse Effects. Epidemiol Rev 2017; 39: 71 - 92. doi:10.1093/epirev/mxx007

[3] Schmid D, Leitzmann MF. Association between physical activity and mortality among breast cancer and colorectal cancer survivors: a systematic review and meta-analysis. Ann Oncol 2014; 25: 1293-1311. doi:10.1093/annonc/mdu012

[4] Hojman P. Exercise protects from cancer through regulation of immune function and inflammation. Biochemical Society transactions 2017. doi:10.1042/bst20160466

[5] Cramp F, Daniel J. Exercise for the management of cancer-related fatigue in adults. The Cochrane database of systematic reviews 2008. doi:10.1002/14651858.CD006145.pub2
[6] Mustian KM, Alfano CM, Heckler C et al. Comparison of Pharmaceutical, Psychological, and Exercise Treatments for Cancer-Related Fatigue: A Meta-analysis. JAMA Oncol 2017; 3: 961 - 968. doi:10.1001/jamaoncol.2016.6914

[7] Schmidt ME, Wiskemann J, Armbrust $P$ et al. Effects of resistance exercise on fatigue and quality of life in breast cancer patients undergoing adjuvant chemotherapy: A randomized controlled trial. Int J Cancer 2015; 137: 471 - 480. doi:10.1002/ijc.29383

[8] Steindorf K, Schmidt ME, Klassen O et al. Randomized, controlled trial of resistance training in breast cancer patients receiving adjuvant radiotherapy: results on cancer-related fatigue and quality of life. Ann Oncol 2014; 25: 2237-2243. doi:10.1093/annonc/mdu374

[9] Baumann FT, Zopf EM, Bloch W. Clinical exercise interventions in prostate cancer patients-a systematic review of randomized controlled trials. Support Care Cancer 2012; 20: 221 -233. doi:10.1007/s00520-011-1271-0

[10] Centemero A, Rigatti L, Giraudo D et al. Preoperative pelvic floor muscle exercise for early continence after radical prostatectomy: a randomised controlled study. Eur Urol 2010; 57: 1039 - 1043. doi:10.1016/j.eururo.2010.02.028

[11] Rogan S, Taeymans J, Luginbuehl $\mathrm{H}$ et al. Therapy modalities to reduce lymphoedema in female breast cancer patients: a systematic review and meta-analysis. Breast Cancer Res Treat 2016; 159: 1 - 14. doi:10.1007| s10549-016-3919-4

[12] Streckmann F, Zopf EM, Lehmann HC et al. Exercise intervention studies in patients with peripheral neuropathy: a systematic review. Sports Med 2014; 44: 1289-1304. doi:10.1007/s40279-014-0207-5

[13] Zimmer P, Baumann FT, Oberste $M$ et al. Effects of Exercise Interventions and Physical Activity Behavior on Cancer Related Cognitive Impairments: A Systematic Review. BioMed Research Int 2016; 2016 : 1820954. doi: 10.1155/2016/1820954

[14] Fornusek CP, Kilbreath SL. Exercise for improving bone health in women treated for stages I-III breast cancer: a systematic review and meta-analyses. J Cancer Surviv 2017; 11 (5): 525 - 541. doi:10.1007/s11764-017-0622-3

[15] Steindorf K, Wiskemann J, Ulrich CM et al. Effects of exercise on sleep problems in breast cancer patients receiving radiotherapy: a randomized clinical trial. Breast Cancer Res Treat 2017; 162: 489-499. doi:10.1007/ s10549-017-4141-8

[16] Buffart LM, Kalter J, Sweegers MG et al. Effects and moderators of exercise on quality of life and physical function in patients with cancer: An individual patient data meta-analysis of 34 RCTs. Cancer Treat Rev 2017; 52: 91 - 104. doi:10.1016/j.ctrv.2016.11.010

[17] Mishra SI, Scherer RW, Snyder C et al. Exercise interventions on healthrelated quality of life for people with cancer during active treatment. The Cochrane database of systematic reviews 2012; 8: CD008465. doi:10.1002/14651858.CD008465.pub2

[18] Mishra SI, Scherer RW, Geigle PM et al. Exercise interventions on healthrelated quality of life for cancer survivors. The Cochrane database of systematic reviews 2012; 8: CD007566 10.1002/14651858.CD007566. pub2

[19] Schmitz KH, Courneya KS, Matthews C et al. American College of Sports Medicine roundtable on exercise guidelines for cancer survivors. Med Sci Sports Exerc 2010; 42: 1409-1426. doi:10.1249/ MSS.0b013e3181e0c112

[20] Winningham ML, MacVicar MG, Burke CA. Exercise for Cancer Patients: Guidelines and Precautions. Phys Sportsmed 1986; 14: 125-134. doi:10.1080/00913847.1986.11709201

[21] Elter T, Stipanov M, Heuser E et al. Is physical exercise possible in patients with critical cytopenia undergoing intensive chemotherapy for acute leukaemia or aggressive lymphoma? Int J Hematol 2009; 90: 199204. doi:10.1007/s12185-009-0376-4

[22] Riemann JF, Ebert M. Positionspapier: Sport (Bewegung) und (Darm-) Krebs. Forum 2017; 32: 187 -191. doi:10.1007/s12312-017-0250 